\title{
Laparoendoscopic single-site (LESS) sacrocolpopexy: feasibility and efficacy of knotless procedure performed with conventional instruments
}

\author{
Andre Luiz Farinhas Tome $\cdot$ Marcos Tobias-Machado • \\ Walter Fernandes Correa
}

Received: 15 September 2010 / Accepted: 14 January 2011 /Published online: 6 April 2011

(C) The Author(s) 2011. This article is published with open access at Springerlink.com

\begin{abstract}
The aim of this paper was to report a case of a patient with stage IV vaginal vault prolapse treated by laparoendoscopic single-site (LESS) sacrocolpopexy using an Alexis retractor and a surgical glove attached to three trocars through a $3.5-\mathrm{cm}$ umbilical incision. Only conventional laparoscopic instruments were used for intrabdominal dissection of vagina and peritoneum. The mesh was fixed to the vaginal fornix and to the sacral periosteum from the promontory using running sutures hold in the extremities by polymer clips. The posterior peritoneum was closed over the mesh. LESS sacrocolpopexy performed with conventional instruments is a difficult but feasible and efficient technique to treat vaginal vault prolapse that respects the principles of conventional laparoscopic or open repairs. Alexis retractor associated with knotless sutures are technical options that simplify LESS reconstructive surgical maneuvers.
\end{abstract}

Keywords Laparoendocopic single-site surgery .

Sacrocolpopexy $\cdot$ Vaginal vault prolapse

\section{Abbreviations \\ LESS Laparoendoscopic single-site \\ POP-Q Pelvic organ prolapse quantification}

A. L. F. Tome $\cdot$ M. Tobias-Machado $\cdot$ W. F. Correa Department of Urology, ABC Medical School, Santo Andre, Brazil
A. L. F. Tome ( $\square)$
Disciplina de Urologia-Faculdade
de Medicina do ABC-Fundacao ABC,
Av. Principe de Gales, 821-Anexo II,
CEP 09060-650 Santo Andre, SP, Brazil
e-mail: andre.tome@sbu.org.br

\section{Introduction}

Vaginal vault prolapse repair relies on either the use of patient's tissue or synthetic materials and can be carried out abdominally or vaginally. Sacrospinous fixation and abdominal sacrocolpopexy are the commonly performed procedures, and the literature is in favor of the second due to its reported higher success rate [1].

Laparoendoscopic single-site (LESS) is a new surgical arena in the development for a great number of indications for urological procedures.

We report a case of complete vaginal vault prolapse which was treated with polypropylene mesh by LESS sacrocolpopexy with conventional laparoscopic instruments.

\section{Case report}

A 52-year-old white woman presented with a complete vaginal vault prolapse for 4 years. Five years before, she had been treated by vaginal hysterectomy due to uterine myomatosis. After 1 year, she developed stress urinary incontinence and was submitted to transobturatory sling and perineoplasty. Another year after, the patient demonstrated a complete vaginal vault prolapse on physical examination (stage IV based on point $\mathrm{C}$ of the pelvic organ prolapse quantification, POP-Q).

The risks and benefits of the surgery were explained to the patient, and she was asked to sign an informed consent approved by institutional review board.

She was treated by $3.5-\mathrm{cm}$ transumbilical laparoendoscopic single-site sacrocolpopexy using an Alexis wound retractor XS ${ }^{\mathrm{TM}}$ (Applied Medical, Rancho Santa Margarita, CA, USA). A surgical glove was attached using three trocars (two of $10 \mathrm{~mm}$ and one of $5 \mathrm{~mm}$ ) that were fixed to 
the outer ring of the wound retractor as previously described (Fig. 1). Patient was positioned in dorsal lithotomy and a Y-shaped polypropylene mesh was passed through the trocar. Only conventional laparoscopic instruments and a $0^{\circ}$ laparoscope were used for intrabdominal dissection of vagina and peritoneum. To achieve a good surgical field, sigmoid colon was retracted laterally by an external suture applied in epiploic appendix. A valve was inserted into the vagina to help the dissection of the posterior peritoneum between vagina and rectum. It was made a mesh with a "Y" format for adequate fixation on the anterior and posterior vaginal wall and the sacrum, with enough length to reach the pelvic floor. The mesh was fixed to the vaginal fornix using two continuous sutures (anterior and apical in vaginal wall) hold in extremities by polymer clips (Hem-o-lok ${ }^{\mathrm{TM}}$; Weck, Research Triangle Park, NC). An external percutaneous suprapubic retraction suture in the mesh helped to achieve good presentation for posterior vaginal suture. The last helical suture was fixed by polymeric clips to the sacral periosteum from the promontory to achieve good vaginal positioning without tension (Fig. 2). The posterior peritoneum was closed over the mesh. The rectopexy is possible, but was not performed.

A second generation cephalosporin was administered immediately before the surgery and was supported by 7 days. The total operative time was $2.5 \mathrm{~h}$. Blood loss was less than $100 \mathrm{~mL}$. Postoperative hospital stay was $18 \mathrm{~h}$. The

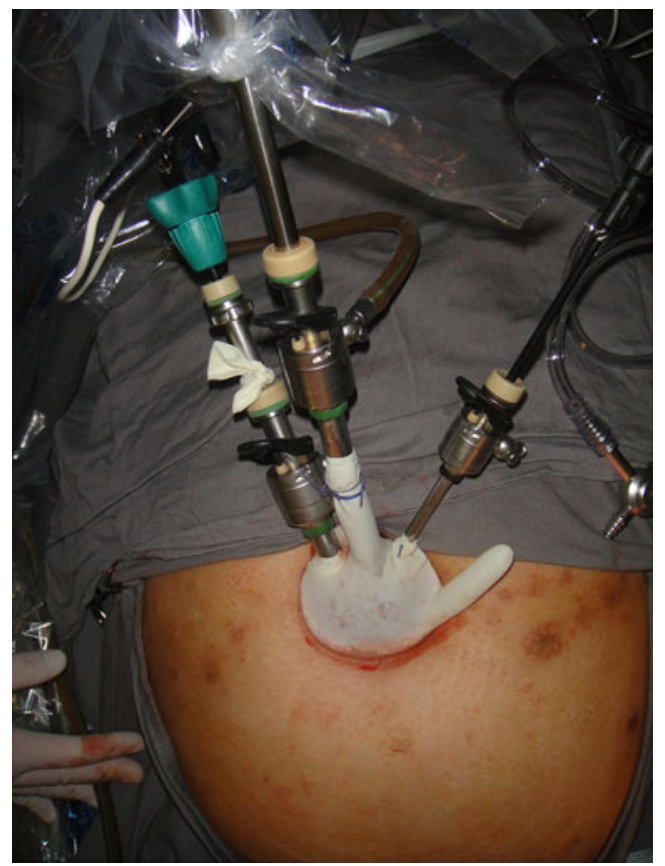

Fig. 1 Transumbilical laparoendoscopic single-site sacrocolpopexy using an Alexis wound retractor XS ${ }^{\mathrm{TM}}$. A surgical glove was attached using three trocars (two of $10 \mathrm{~mm}$ and one of $5 \mathrm{~mm}$ ) that were fixed to the outer ring of the wound retractor

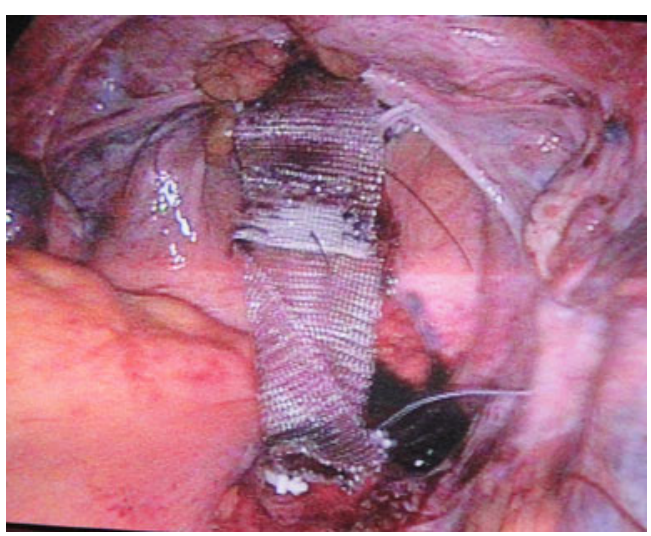

Fig. 2 The mesh was fixed to the vaginal fornix using two continuous sutures (anterior and apical in vaginal wall) hold in extremities by polymer clips. The last helical suture was fixed by polymeric clips to the sacral periosteum from the promontory to achieve good vaginal positioning without tension

patient was referred to the outpatient clinic 1 week postoperatively. During this visit, no complications including wound infections, intra-abdominal abscess formation, and urinary incontinence were observed. The patient became very satisfied with her surgical scar and with the functional result after a 3month follow-up. The surgery resulted in an apical anatomic success (stage 0 of the POP-Q).

\section{Discussion}

Mesh procedures are gaining in popularity, and preliminary data from this technique for correcting vaginal vault is encouraging. As the aging population increases, the incidence of prolapse will also rise, older techniques using native tissue will continue, while new techniques using the mesh need to be studied further [1].

A systematic review evaluated the efficacy and safety of mesh in surgery for uterine or vault prolapse using 54 studies involving 7,054 women. For sacrocolpopexy (average follow-up 23 months), the risk of clinical recurrence ranged from $0 \%$ to $6 \%$, persistent symptoms from $3 \%$ to $31 \%$, and mesh erosion from $0 \%$ to $12 \%$ [2].

Comparing minimally invasive approaches, there are preliminary evidences that laparoscopy offers some advantages over vaginal repair. Descargues et al. compared the safety and the efficacy of the laparoscopic and vaginal technique for the surgical management of pelvic organ prolapse. The tolerance of prostheses by laparoscopy is safe. The biological prostheses, introduced vaginally, offer the same advantages. The anatomical results in the medium term (30 months) seem more favorable to laparoscopy than transvaginal approach, as well as functional results but they still need to better evaluated [3]. 
Single-port laparoscopy, also known as LESS surgery, has emerged as an attempt to further enhance cosmetic benefits and reduce morbidity of minimally invasive surgery. Refinement and modification of laparoscopic instrumentation, such as preventing or actively articulating instruments, have resulted in a substantial increase in the description of LESS in urology over the past 2 years. Technical feasibility has been demonstrated for a broad range of extirpative and reconstructive procedures. However, most comparative studies for other indications observed better esthetic results with no advantage regarding operative and recovery parameters. We find only one series of 13 cases of LESS sacrocolpopexy in literature and the authors showed comparable efficacy and superior cosmetics compared to alternative approaches [4].

LESS surgery has many technical challenges, such as loss of triangulation and tissue manipulation, internal and external clashing of instruments and handles, and visualization of the structures in a more medial perspective. The technique we describe in this article attempts to reproduce the principles of the conventional repair associated with facilitative maneuvers, using only conventional instruments. The use of the Alexis wound retractor provides atraumatic circumferential retraction of the fascial opening. The glove expands with insufflation providing additional distance among the instruments, better triangulation, lesser clash, and greater freedom of movement for instruments. This homemade single-port device, first described by Ryu [5], also has the advantage to use freely the number and the size of the ports, associated with the lower cost when compared to the purpose-built multichannel ports. The Alexis consists of one inner ring, an outer ring, and a cylindrical plastic sleeve. The inner ring was introduced through the umbilical incision into the peritoneal cavity. The cylindrical plastic sleeve was then pulled outward to cinch the inner ring toward the parietal wall and furling the outer ring and the plastic sleeve outward. As the outer ring was turned inside out, a latex surgical glove was adapted to it and continued furling together until the sleeve had created complete tension. This incremental retraction and furling of the sleeve drew the inner and outer rings together on either side of the abdominal wall, thereby creating tension in the sleeve between the rings that retracts the edges of the abdominal wall apart atraumatically, and also providing an effective seal, preventing gas leakage. The tips of the second, third, and fourth finger of the latex surgical glove were excised, a 10-mm trocar was inserted through the middle finger for the laparoscope and gas insufflation, and $10-\mathrm{mm}$ and $5-\mathrm{mm}$ trocars were inserted at the second and the fourth finger for conventional rigid laparoscopic instruments, respectively, and tied tightly to the glove using 2-0 cotton.

We performed a knotless technique, using Hem-o-lok clips in the extremities of the sutures to facilitate the reconstruction. This option is especially useful in LESS procedures when there are no articulated needle holders or graspers due to acute angulation among the instruments. The use of Hem-o-lok had been already described during laparoscopic partial nephrectomy. This technique makes it much easier to suture. There was no sign of erosion of the vaginal wall or complaint of dyspareunia. Extracorporeal knots are really an option for the suture.

LESS sacrocolpopexy is a technique in its infancy. Further prospective studies comparing long-term results to standard laparoscopy are awaited to indicate the real application of LESS sacrocolpopexy in the modern urological armamentarium. We believe that LESS represents a new provocative step forward in the arena of minimally invasive surgery.

Conflicts of interest None.

Open Access This article is distributed under the terms of the Creative Commons Attribution Noncommercial License which permits any noncommercial use, distribution, and reproduction in any medium, provided the original author(s) and source are credited.

\section{References}

1. Uzoma A, Farag KA (2009) Vaginal vault prolapse. Obstet Gynecol Int 2009:275621

2. Jia X, Glazener C, Mowatt G, Jenkinson D, Fraser C, Bain C et al (2010) Systematic review of the efficacy and safety of using mesh in surgery for uterine or vaginal vault prolapsed. Int Urogynecol J Pelvic Floor Dysfunct (in press)

3. Descargues G, Collard P, Grise P (2008) Surgical management of pelvic organ prolapse in women: laparoscopic or vaginal sacrocolpopexy? Gynécol Obstét Fertil 36(10):978-983

4. White WM, Goel RK, Swartz MA, Moore C, Rackley RR, Kaouk JH (2009) Single-port laparoscopic abdominal sacral colpopexy: initial experience and comparative outcomes. Urology 74(5):10081012

5. Ryu DS, Park WJ, Oh TH (2009) Retroperitoneal laparoendoscopic single-site surgery in urology: initial experience. J Endourol 23 (11):1857-1862 